\title{
CUBE COMPLEXES, SUBGROUPS OF MAPPING CLASS GROUPS, AND NILPOTENT GENUS
}

\author{
MARTIN R. BRIDSON
}

\section{INTRODUCTION}

These notes are based on my lecture at PCMI in July 2012. They are structured around two sets of results, one concerning groups of automorphisms of surfaces and the other concerning the nilpotent genus of groups. The first set of results exemplifies the theme that even the nicest of groups can harbour a diverse array of complicated finitely presented subgroups: we shall see that the finitely presented subgroups of the mapping class groups of surfaces of finite type can be much wilder than had been previously recognised. The second set of results fits into the quest to understand which properties of a finitely generated group can be detected by examining the group's finite and nilpotent quotients and which cannot.

These two topics appear to have little in common and neither has any obvious connection to the study of non-positively curved cube complexes; they are chosen for exactly these reasons. My purpose is to describe the resolution of various long-standing problems in a way that emphasizes the broad applicability of a certain template for constructing interesting examples of finitely presented groups. This template, described in Section 8, can be applied in many other contexts. It refines a construction that I articulated in [17], with improvements based on recent advances in the understanding of right-angled Artin groups (RAAGS) and non-positively curved cube complexes (particularly the virtually special cube complexes of Haglund and Wise).

The new results concerning subgroups of mapping class groups are from [16] while the new results concerning nilpotent genera of groups are from [29] (which is part of a wider project with Alan Reid from the University of Texas). Two of the results that we shall discuss are the following.

Theorem 1.1. [16] If the genus of a surface $S$ is sufficiently large, then the isomorphism problem for the finitely presented subgroups of the mapping class group $\operatorname{Mod}(S)$ is unsolvable.

Theorem 1.2. [29] There exist pairs of finitely generated, residually torsion-free-nilpotent groups $N \hookrightarrow \Gamma$ so that $N$ has the same finite and nilpotent quotients as $\Gamma$ but $\Gamma$ is finitely presented while $\mathrm{H}_{2}(N, \mathbb{Q})$ is infinite dimensional.

Date: final edits, Sat 12 Oct 2013.

1991 Mathematics Subject Classification. 20F65, 57M60, 20E26 (20F36, 20F67, 20F10).

Key words and phrases. Mapping class groups, RAAGs, cube complexes, nilpotent genus, decision problems. 
Several of the results that we shall discuss concern decision problems for groups. I shall assume that the reader is familiar with the basic vocabulary associated to such problems, and recommend [56] as a pleasant introduction to the subject.

\section{SUbGROUPS OF MAPPING CLASS GROUPS}

Throughout, $S$ will denote a compact, connected surface, which is allowed to have non-empty boundary. Most of the results that we will discuss remain valid for compact surfaces with finitely many punctures. The mapping class group of $S$ will be denoted ${ }^{1}$ $\operatorname{Mod}(S)$; this is the group of isotopy classes of orientation-preserving homeomorphisms $S \rightarrow S$, where isotopies are required to fix the boundary point-wise. I shall assume that the reader is familiar with the basic ideas and vocabulary concerning mapping class groups, as described in [42] and [50] for example.

2.1. The first subgroups. Cyclic subgroups: Nielsen-Thurston theory describes the individual elements of $\operatorname{Mod}(S)$ : an element $\phi \in \operatorname{Mod}(S)$ is reducible if it is the class of a homeomorphism that leaves invariant a non-empty collection of homotopically-essential circles on $S$, none of which is homotopic to a boundary component; Thurston proved that the irreducible elements $\psi$ of infinite order are pseudo-Anosov, which implies in particular that if $c$ is a loop that is not homotopic into $\partial S$ then, in any fixed metric, the length of the shortest loop in the homotopy class $\psi^{n}[c]$ grows exponentially with $|n|$.

Nielsen proved that the elements of finite order in $\operatorname{Mod}(S)$ are precisely those mapping classes which contain a diffeomorphism $f$ such that $f^{d}=\operatorname{id}_{S}$ for some $d$.

Finite subgroups: Kerckhoff's resolution of the Nielsen Realisation Problem [51] shows that every finite subgroup $G<\operatorname{Mod}(S)$ arises as a group of isometries of a metric of constant curvature on $S$; equivalently, $G$ has a fixed point in the natural action of $\operatorname{Mod}(S)$ on the Teichmüller space $\mathcal{T}(S)$. The action of $\operatorname{Mod}(S)$ on $\mathcal{T}(S)$ is proper and there is an equivariant retraction onto a spine where the action of $\operatorname{Mod}(S)$ is cocompact [59]. It follows that there are only finitely many conjugacy classes of finite subgroups in $\operatorname{Mod}(S)$; see [20]. This does not remain true if one $\operatorname{replaces} \operatorname{Mod}(S)$ by a finitely presented subgroup (Theorem 2.4).

Abelian subgroups: The Dehn twists in disjoint curves on $S$ have infinite order and commute. On a closed surface of genus $g$ the maximum number of disjoint, non-homotopic, essential simple closed curves that one can fit is $3 g-3$; if $S$ has $b$ boundary components then one can add a further $b$ curves parallel to the boundary components. Thus we obtain free abelian groups of rank $3 g-3+b$ generated by Dehn twists. Birman-LubotzkyMcCarthy [14] proved that every abelian subgroup of $\operatorname{Mod}(S)$ is finitely generated and has rank no greater than $3 g-3+b$ (see also [50]).

Free subgroups: These abound in $\operatorname{Mod}(S)$, indeed Ivanov ${ }^{2}$ [50] and McCarthy [54] proved that mapping class groups satisfy a Tits Alternative: if a subgroup $G<\operatorname{Mod}(S)$ is not virtually abelian, then it contains a free subgroup. More explicitly, if $\phi, \psi \in \operatorname{Mod}(S)$ are pseudo-Anosov, then for some $n>0$, the subgroup $\left\langle\phi^{n}, \psi^{n}\right\rangle$ is free (and cyclic only if $\phi$ and $\psi$ share an axis in Teichmüller space).

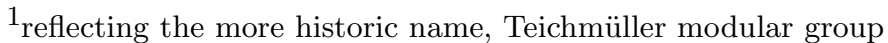

${ }^{2}$ Most of the results of Ivanov that I quote were proved in his earlier papers, but [50] provides an excellent, coherent account of his work.
} 
A more involved argument due to Dahmani, Guirardel and Osin [36] shows that if $\psi \in \operatorname{Mod}(S)$ is pseudo-Anosov, then for suitable $m>0$, the normal closure $\left\langle\left\langle\psi^{m}\right\rangle\right\rangle$ is a free group freely generated by the conjugates of $\psi^{m}$.

Direct Products: If $S^{\prime}$ and $S^{\prime \prime}$ are disjoint subsurfaces of $S$, then by extending diffeomorphisms to be the identity on the complement we see that $\operatorname{Mod}\left(S^{\prime}\right) \times \operatorname{Mod}\left(S^{\prime \prime}\right)$ is a subgroup of $\operatorname{Mod}(S)$.

2.2. Wreath products. The wreath product $A \nmid B$ of groups is the semidirect product $B \ltimes \oplus_{b \in B} A_{b}$, where the $A_{b}$ are isomorphic copies of $A$ permuted by left translation.

Proposition 2.1. If $S$ is a compact surface with non-empty boundary and $G$ is a finite group, then there is a closed surface $S_{g}$ and a monomorphism $\operatorname{Mod}(S) \imath G \rightarrow \operatorname{Mod}\left(S_{g}\right)$.

To prove this, one first reduces to the case where $S$ has one boundary component. Then one takes a closed surface on which $G$ acts effectively, deletes a family of open discs centred at the points of a free $G$-orbit, and attaches a copy of $S$ to each of the resulting boundary circles, extending homeomorphisms of each copy of $S$ by the identity on the complement. (See [18] for details.)

For a group $H$ with finite-index $K \triangleleft H$, there is a standard embedding $H \hookrightarrow K$ ? $(H / K)$.

Corollary 2.2. If a group $H$ has a subgroup of finite index that embeds in the mapping class group of a compact surface with boundary, then $H$ embeds in the mapping class group of a closed surface.

Remark 2.3. There is considerable flexibility in the above construction, but cannot hope to embed $H$ in $\operatorname{Mod}(S)$ for all surfaces of sufficiently high genus. Indeed there are constraints even for finite groups [53].

2.3. Non-subgroups. Reducibility and its Consequences. The Reduction Theory of Ivanov [50] allows one to prove all manner of results concerning the subgroups of mapping class groups by induction on the complexity of the surface. To explain this, we first extend the definition of reducibility to subgroups: $G<\operatorname{Mod}(S)$ is reducible if it leaves invariant a non-empty collection of homotopically-essential circles on $S$, none of which is homotopic to a boundary component. Ivanov [50] proves that if $G<\operatorname{Mod}(S)$ is infinite and irreducible then it contains a pseudo-Anosov element.

There are subgroups of finite index $P<\operatorname{Mod}(S)$ in the mapping class that consist entirely of pure automorphisms: $[\phi]$ is pure if, on the complement of a tubular neighbourhood of the set of disjoint curves that $\phi$ fixes, the restriction of $\phi$ to each component is either trivial or pseudo-Anosov. The kernel of the action of $\operatorname{Mod}(S)$ on $H_{1}\left(S, \mathbb{Z}_{3}\right)$ is pure, for example [50, p.4]. With this fact in hand, it follows from Ivanov's theorem that every subgroup $G<\operatorname{Mod}(S)$ fits into a short exact sequence where the kernel (which is central) is a free abelian group generated by multi-twists in the reducing curves for $G$ and the quotient is a direct product of subgroups of mapping class groups of smaller surfaces, each containing a pseudo-Anosov on that surface.

From there, it is not difficult to prove, for example, that every amenable subgroup is abelian (cf. [50], [14]). And using [36] one sees that $\operatorname{Mod}(S)$ does not contain infinite images of groups that are $\mathbb{Z}$-averse in the sense of [30], from which it follows that 
all homomorophisms to $\operatorname{Mod}(S)$ from irreducible lattices in higher-rank semisimple Lie groups have finite image (cf. [43]).

Other obstructions to embedding come from global properties of $\operatorname{Mod}(S)$ that are inherited by subgroups; for example, if $H<\operatorname{Mod}(S)$ then $H$ must be residually-finite [44], and all cyclic subgroups of $H$ must be quasi-isometrically embedded [41].

2.4. Subgroups that are not finitely presented. We are concerned here almost entirely with subgroups that are finitely presented, or at least finitely generated. But it would be remiss of me not to mention that $\operatorname{Mod}(S)$ has a host of natural subgroups where these finiteness conditions may fail. Foremost among these is the Torelli group, which is the kernel of the action of $\operatorname{Mod}(S)$ on $H_{1}(S, \mathbb{Z})$. This is finitely generated if the genus of $S$ is at least 3 but it is not known if it is finitely presented. The kernel of the action $\operatorname{Mod}(S)$ on $\pi_{1}(S)$ modulo any later term of the lower central series is not even finitely generated. Connecting to the second part of this talk, we note that the Torelli group is residually torsion-free nilpotent [5].

2.5. On the difficulty of identifying finitely presented subgroups. For the most part, the heart of geometric and combinatorial group theory lies with the study of finitely presented groups, but this restriction brings with it real challenges. In any context, it is easy to find finitely generated subgroups of a given group $G$ : one can simply take a finite subset $S \subset G$ and consider $\langle S\rangle$. But typically there will be no algorithm to decide which finite subsets of $G$ generate finitely presentable subgroups (this is the case in a direct product of free groups already). Moreover, even if one is given information that guarantees that $\langle S\rangle$ has a finite presentation, there is no general procedure that will produce such a presentation, even when the ambient group is as benign as $G=\mathrm{GL}(n, \mathbb{Z})$; see [30].

2.6. Direct products of free groups. If $H_{1}, H_{2}<\operatorname{Mod}(S)$ are supported on disjoint subsurfaces of $S$, then they commute. One can embed $g$ disjoint one-holed tori in a surface of genus $g$, and the mapping class group of a one-holed torus is a central extension of $\mathrm{SL}(2, \mathbb{Z})$, which contains non-abelian free groups. Thus, if $S$ has genus $g$ (and any number of boundary components), then $\operatorname{Mod}(S)$ contains the direct product $D$ of $g$ non-abelian free groups. In $D$, what (finitely generated or finitely presented) subgroups might we find? The answer to this question leads us in the main direction of this lecture. But first we pause to record a consequence of Corollary 2.2.

Theorem 2.4. There exist closed surfaces $S$ and finitely presented subgroups $H<$ $\operatorname{Mod}(S)$ such that $H$ has infinitely many conjugacy classes of elements of finite order.

Proof: The direct product $\Gamma$ of $n$ copies of $\mathrm{SL}(2, \mathbb{Z})$ contains as a subgroup of finite index the direct product $D$ of $n$ non-abelian free groups. $D$ embeds in the mapping class group of a surface of genus $n$ with 1 boundary component, so $\Gamma$ embeds in $\operatorname{Mod}(S)$ for some closed surface $S$. It is proved in [20] that $\Gamma$ contains finitely presented subgroups with infinitely many conjugacy classes of elements of order 4. (See also [15].)

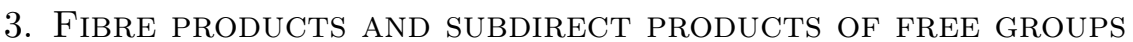

We shall develop our discussion of these subgroups around two constructions. 
Construction 3.1. Fibre Products. Let $Q=\langle A \mid R\rangle$ be a finitely presented group. Let $F$ be the free group on $A$ and let $p: F \rightarrow Q$ be the surjection implicit in the notation. The kernel of $p$ is finitely generated if and only if $Q$ is finite, but regardless of what $Q$ is, the fibre product

$$
P=\{(u, v) \mid p(u)=p(v)\}<F \times F
$$

will be generated by the finite set $\Sigma_{Q}=\{(a, a),(r, 1) ; a \in A, r \in R\}$.

This observation provides complicated finitely generated subgroups of $F \times F$ : Mihailova [57] and Miller [55] were the first to see if $Q$ has an unsolvable word problem, then $P$ has an unsolvable conjugacy problem (cf. Proposition 7.6) and there no algorithm to decide which words in the generators of $F \times F$ define elements of $P$ (Proposition 7.5); and since $P \cong F \times F$ if and only if $Q=1$, there can be no algorithm to decide isomorphism among the finitely generated subgroups of $F \times F$, because there is no algorithm that can determine which finitely presented groups (with generating sets of a fixed cardinality) are trivial. There isn't even an algorithm that, given a finite subset $\Sigma \subset F \times F$, can calculate the first homology of $\langle\Sigma\rangle$ (see [27]).

There are uncountably many 2-generator groups, and via fibre products one can deduce from this that there are uncountably many non-isomorphic subgroups $P<F \times F$ (see [10]). Hence:

Proposition 3.2. The mapping class group of any surface of genus at least 2 contains uncountably many non-isomorphic subgroups.

These fibre products, though, do not give us complicated finitely presented subgroups of mapping class groups, because $P$ is finitely presentable if and only if $Q$ is finite [45]. Indeed, Baumslag and Roseblade [10] proved that $F \times F$ has no finitely presented subgroups other than the obvious ones: if $G<F \times F$ is finitely presented, then $G$ is either free or else it has a subgroup of finite index that is a product of two free groups (its intersections with the direct factors).

3.1. Finitely presented examples. A celebrated construction of Stallings [65] and Bieri [12] shows that there are interesting finitely presented subgroups in the direct product of three (or more) free groups.

Construction 3.3. The Stallings-Bieri Groups. Let $h: F \times \cdots \times F \rightarrow \mathbb{Z}$ be a homomorphism that restricts to an epimorphism on each of the $n$ factors. Stallings [65] (in the case $n=3$ ) and Bieri [12] proved that the kernel $\mathrm{SB}_{n}$ has a classifying space with a finite $(n-1)$-skeleton, but $H_{n}\left(\mathrm{SB}_{n}, \mathbb{Z}\right)$ is not finitely generated.

In the light of the discussion in Construction 3.1, one might anticipate that these examples are the tip of an iceberg of pathology akin to the wildness that we saw among the finitely generated subgroups of $F \times F$. But Bridson and Miller [28] (cf. [25]) proved, roughly speaking, that variations on the construction of Stallings and Bieri account for all the finitely presented subdirect products of free groups. Recall that a subgroup of a direct product $H<G_{1} \times \cdots \times G_{d}$ is termed a subdirect product if its projection to each of the factors is onto, and $H$ is said to be full if all of the intersections $H \cap G_{i}$ are non-trivial. 
Theorem 3.4. [28] If $H<F_{1} \times \cdots \times F_{n}=D$ is a full subdirect product of finitely generated free groups, then there is a subgroup of finite index $D_{0}<D$ such that $H$ contains the $(n-1)$ st term of the lower central series of $D_{0}$.

\section{A NEW LEVEL OF COMPLICATION}

We now come to the cluster of new results concerning subgroups of mapping class groups. These theorems show that the finitely presented subgroups of mapping class groups can be vastly more complicated than those studied hitherto. The proofs of these theorems will be outlines in later sections, where they are used to illustrate a general technique for constructing wild subgroups in varied contexts (which is the main theme of this lecture).

Readers unfamiliar with decision problems may wish to consult [56]. For an account of the history of the problems settled by the following theorems, see [40].

Theorem 4.1. [16] If the genus of $S$ is sufficiently large, then the isomorphism problem for the finitely presented subgroups of $\operatorname{Mod}(S)$ is unsolvable.

In more detail, there is a recursive sequence $\Delta_{i}(i \in \mathbb{N})$ of finite subsets of $\operatorname{Mod}(S)$, together with finite presentations $\left\langle\Delta_{i} \mid \Theta_{i}\right\rangle$ of the subgroups they generate, such that there is no algorithm that can determine whether or not $\left\langle\Delta_{i} \mid \Theta_{i}\right\rangle \cong\left\langle\Delta_{0} \mid \Theta_{0}\right\rangle$.

Theorem 4.2. [16] If the genus of $S$ is sufficiently large, then there is a finitely presented subgroup of $\operatorname{Mod}(S)$ with unsolvable conjugacy problem.

Theorem 4.3. [16] If the genus of $S$ is sufficiently large, then there are finitely presented subgroups of $\operatorname{Mod}(S)$ for which the membership problem is unsolvable.

The Dehn function of a finitely presented group $\Gamma=\langle A \mid R\rangle$ estimates the complexity of the word problem by counting the number of times one has to apply the defining relations in order to prove that a word $w$ in the generators represents the identity in the group: $\operatorname{Area}(w)$ is defined to be the least integer $N$ for which there is an equality

$$
w=\prod_{i=1}^{N} \theta_{i} r_{i}^{ \pm 1} \theta_{i}^{-1}
$$

in the free group $F(A)$, with $r_{i} \in R$, and the Dehn function of $\langle A \mid R\rangle$ is

$$
\delta(n):=\max \left\{\operatorname{Area}(w)\left|w=_{\Gamma} 1,\right| w \mid \leq n\right\},
$$

where $|w|$ denotes word-length. Mapping class groups have quadratic Dehn functions [60], as do the finitely presented subgroups described in the previous sections.

Theorem 4.4. [16] If the genus of $S$ is sufficiently large, then there are finitely presented subgroups of $\operatorname{Mod}(S)$ whose Dehn functions are exponential.

One might hope to prove some of these theorems by focussing on subgroups of direct products of free groups, but restrictions that follow from Theorem 3.4 dash this hope. The following theorem was proved in [28] and extended to all finitely presented subgroups of residually-free groups in [26]. 
Theorem 4.5. [28] The conjugacy problem is solvable for every finitely presented subgroup of a direct product of free (or surface) groups, and there is a uniform solution to the membership problem for all such subgroups.

In Section 6 we consider how we might enlarge the class of direct products of free groups so as to obtain wilder subgroups (via fibre product constructions) while retaining enough geometry to provide embeddings into mapping class groups. But first we turn to a different topic.

\section{The Nilpotent Genus of A GROUP}

This section is based on joint work with Alan Reid from the University of Texas [29].

If each finite subset of a group $\Gamma$ injects into some nilpotent (or finite) quotient of $\Gamma$, then one expects to be able to detect many properties of $\Gamma$ from the totality of its nilpotent (or finite) quotients. Which properties can be detected and which cannot? Forms of this question have stimulated a lot of research into discrete and profinite groups over the last forty years, and there has been a particular resurgence of interest recently, marked by several notable breakthroughs. Here we focus on the nilpotent quotients.

Recall that a group $\Gamma$ is said to be residually nilpotent (resp. residually torsionfree-nilpotent) if for each non-trivial $\gamma \in \Gamma$ there exists a nilpotent (resp. torsion-freenilpotent) group $Q$ and a homomorphism $\phi: \Gamma \rightarrow Q$ with $\phi(\gamma) \neq 1$. Thus $\Gamma$ is residually nilpotent if and only if $\bigcap \Gamma_{n}=1$, where $\Gamma_{n}$ is the $n$-th term of the lower central series of $\Gamma$, defined inductively by setting $\Gamma_{1}:=\Gamma$ and $\Gamma_{n+1}:=\left\langle[x, y]: x \in \Gamma_{n}, y \in \Gamma\right\rangle$.

We say that two residually nilpotent groups $\Gamma$ and $\Lambda$ have the same nilpotent genus if they have the same nilpotent quotients; this is equivalent to requiring that $\Gamma / \Gamma_{c} \cong \Lambda / \Lambda_{c}$ for all $c \geq 1$.

Examples 5.1. Examples of finitely generated residually torsion-free-nilpotent groups include free groups $F_{n}$ (hence residually free groups such as surface groups and limit groups), right-angled Artin groups (RAAGs) [38], the Torelli subgroup of the mapping class group [5], and $\mathrm{IA}_{n}<\operatorname{Out}\left(F_{n}\right)$, the kernel of the natural map $\operatorname{Out}\left(F_{n}\right) \rightarrow \operatorname{GL}(n, \mathbb{Z})$ (see [3], [5], [30]), and the corresponding subgroup in the outer automorphism group of any RAAG [66].

A group is termed parafree if it is residually nilpotent and has the same genus as a free group. The existence of families of parafree groups that are not free gives a first inkling of the diversity that can exist within a fixed nilpotent genus. One such family was discovered by Gilbert Baumslag (see [6]):

$$
G_{i j}=\left\langle a, b, c \mid a=\left[c^{i}, a\right] \cdot\left[c^{j}, b\right]\right\rangle .
$$

In [7] Baumslag surveyed the state of the art concerning groups of the same nilpotent genus and compiled a list of open problems that are of particular importance in the field. Here we shall concentrate on three problems whose resolution will serve to emphasize how different groups within a given genus can be. (Other problems on Baumslag's list challenge the reader to establish commonalities across a genus; cf. [29] Theorem C.)

Problems 5.2. Do there exist pairs of groups of the same nilpotent genus such that

- one is finitely presented and the other is not; or 
- both are finitely presented, one has a solvable conjugacy problem but the other does not; or

- one has finitely generated second homology $\mathrm{H}_{2}(-, \mathbb{Z})$ and the other does not?

These questions are settled by the following compilation of results from [29].

Theorem 5.3. (1) There exist pairs of finitely presented, residually torsion-freenilpotent groups $P \hookrightarrow \Gamma$ of the same nilpotent genus such that $\Gamma$ has a solvable conjugacy problem and $P$ does not.

(2) There exist pairs of finitely generated, residually torsion-free-nilpotent groups $N \hookrightarrow \Gamma$ of the same nilpotent genus such that $\Gamma$ is finitely presented while $\mathrm{H}_{2}(\mathrm{~N}, \mathbb{Q})$ is infinite dimensional (so in particular $\mathrm{N}$ is not finitely presented).

Remark 5.4. The pairs of groups that are constructed in [29] to prove this theorem have the additional property the inclusion map induces an isomorphism of profinite and pro-nilpotent completions.

5.1. Criteria for pro-nilpotent equivalence. The following theorem of John Stallings [64] provides a useful criterion for establishing that groups have the same nilpotent genus: If a homomorphism of groups $u: N \rightarrow \Gamma$ induces an isomorphism on $H_{1}(-, \mathbb{Z})$ and an epimorphism on $H_{2}(-, \mathbb{Z})$, then $u_{c}: N / N_{c} \rightarrow \Gamma / \Gamma_{c}$ is an isomorphism for all $c \geq 1$.

Given a short exact sequence of groups $1 \rightarrow N \rightarrow G \rightarrow Q \rightarrow 1$, the LyndonHochschild-Serre (LHS) spectral sequence (which is explained on page 171 of [31]) calculates the homology of $G$ in terms of the homology $N$ and $Q$. The terms on the $E^{2}$ page of the spectral sequence are $E_{p q}^{2}=H_{p}\left(Q, H_{q}(N, \mathbb{Z})\right)$, where the action of $Q$ on $H_{*}(N, \mathbb{Z})$ is induced by the action of $G$ on $N$ by conjugation. The following proposition is proved in [29] by using this spectral sequence to see that $U \rightarrow G$ satisfies the hypotheses of Stallings' theorem.

Proposition 5.5. Let $1 \rightarrow N \stackrel{u}{\rightarrow} \Gamma \rightarrow Q \rightarrow 1$ be a short exact sequence of groups and let $u_{c}: N / N_{c} \rightarrow \Gamma / \Gamma_{c}$ be the homomorphism induced by $u: N \hookrightarrow \Gamma$. Suppose that $N$ is finitely generated, that $Q$ has no non-trivial finite quotients, and that $H_{2}(Q, \mathbb{Z})=0$. Then $u_{c}$ is an isomorphism for all $c \geq 1$. In particular, if $\Gamma$ is residually nilpotent then $N$ and $\Gamma$ have the same nilpotent genus.

Corollary 5.6. Under the hypotheses of Proposition 5.5, the inclusion $P \hookrightarrow \Gamma \times \Gamma$ of the fibre product induces an isomorphism $P / P_{c} \rightarrow \Gamma_{c} \times \Gamma_{c}$ for every $c \in \mathbb{N}$ and hence an isomorphism $\widehat{P}_{\text {nil }} \rightarrow \widehat{\Gamma}_{\text {nil }} \times \widehat{\Gamma}_{\text {nil }}$.

Proof. We have inclusions $N \times N \stackrel{i}{\rightarrow} P \stackrel{j}{\rightarrow} \Gamma \times \Gamma$ and the proposition implies that $i$ and $j \circ i$ induce isomorphisms modulo any term of the lower central series, and therefore $j$ does as well.

5.2. Relation with profinite genus. Let $\Gamma$ be a finitely generated group. If one orders the normal subgroups of finite index $N<\Gamma$ by reverse inclusion, then the quotients $\Gamma / N$ form an inverse system whose limit

$$
\widehat{\Gamma}=\lim _{\longleftarrow} \Gamma / N
$$


is the profinite completion of $\Gamma$. Similarly, the pro-nilpotent completion, denoted $\widehat{\Gamma}_{\text {nil }}$ is the inverse limit of the nilpotent quotients of $\Gamma$. Every homomorphism of discrete groups $u: H \rightarrow G$ induces a homomorphism $\hat{u}: \widehat{H} \rightarrow \widehat{\Gamma}$ and a homomorphism $\widehat{u}_{\text {nil }}: \widehat{H}_{\text {nil }} \rightarrow \widehat{\Gamma}_{\text {nil }}$.

If $H, G$ are residually nilpotent, they lie in the same nilpotent genus if and only if $\widehat{H}_{\text {nil }} \cong \widehat{G}_{\text {nil }}$. If $G$ is nilpotent then $G=\widehat{G}_{\text {nil }}$.

There are finitely generated nilpotent groups $H \nRightarrow G$ that have the same finite quotients; thus $\widehat{H}=\widehat{G}$ but $\widehat{H}_{\text {nil }} \nRightarrow \widehat{G}_{\text {nil }}$. The situation is quite different if the isomorphism of profinite completions is induced by a homomorphism of the discrete groups.

Proposition 5.7. [29] Let $u: P \hookrightarrow \Gamma$ be a pair of finitely generated, residually finite groups, and for each $c \geq 1$, let $u_{c}: P / P_{c} \rightarrow \Gamma / \Gamma_{c}$ be the induced homomorphism. If $\widehat{u}: \widehat{H} \rightarrow \widehat{\Gamma}$ is an isomorphism, then $u_{c}$ is an isomorphism for all $c \geq 1$, hence $\widehat{P}_{\text {nil }} \cong \widehat{\Gamma}_{\text {nil }}$.

With this observation in hand, we see that (modulo variations in the finiteness assumptions) Proposition 5.5 is a weak form of the following proposition, which originates in the work of Platonov and Tavgen [61] where the first pairs of finitely generated groups $P \hookrightarrow \Gamma$ satisfying the hypotheses of Proposition 5.7 were constructed. (Such pairs of groups are now known as Grothendieck pairs.) This proposition also played an important role in the Bridson-Grunewald construction of Grothendieck pairs where both $P$ and $\Gamma$ are finitely presented [23].

Proposition 5.8. Let $1 \rightarrow N \rightarrow \Gamma \rightarrow Q \rightarrow 1$ be a short exact sequence of groups with $\Gamma$ finitely generated and let $P$ be the associated fibre product. Suppose that $Q \neq 1$ is finitely presented, has no proper subgroups of finite index, and $H_{2}(Q, \mathbb{Z})=0$. Then

(1) $P \rightarrow \Gamma \times \Gamma$ induces an isomorphism $\widehat{P} \rightarrow \widehat{\Gamma}$;

(2) if $N$ is finitely generated then $N \rightarrow \Gamma$ induces an isomorphism $\widehat{N} \rightarrow \widehat{\Gamma \times \Gamma}$.

\section{Cubes, RAAGs and $\operatorname{CAT}(0)$}

In this section we shall see right-angled Artin groups (RAAGs) emerge as a generalisation of direct products of free groups. RAAGs have a similar cubical geometry to $F \times \cdots \times F$ and are residually torsion-free nilpotent; moreover it is easy to get them to act on surfaces. But, crucially for us, they harbour a much greater array of finitely presented subgroups.

We take up the theme of Construction 3.3, retaining the notation.

The original proofs of Stallings and Bieri are essentially algebraic. Bestvina and Brady [11] discovered a geometric proof that motivated their theory of Morse theory for cubical complexes. If we regard $F$ as the fundamental group of a compact simplicial graph $Y$, then $D=F \times \cdots \times F$ is the fundamental group of $X=Y \times \cdots \times Y$, which has a natural cubical structure. This cube complex is non-positively curved in the sense of Alexandrov, i.e. locally CAT(0). (The standard reference for CAT(0) spaces is [24], but the lectures of Caprace and Sageev in this volume cover more than enough for our needs.)

The vertex set of the universal cover is $D$ and the homomorphism $h: D \rightarrow \mathbb{Z}$ can be extended linearly across cells to give a Morse function $\tilde{X} \rightarrow \mathbb{R}$. Bestvina and Brady [11] determine the finiteness properties of the kernel of $h$ by examining the way in which the sublevel sets of this Morse function change as one passes through critical points (vertices). 
They extended this analysis to the larger class of cubical complexes defined below, and in this way settled long-standing questions concerning the relationship between different finiteness properties of groups.

A right angled Artin group (RAAG) is a group given by a presentation of the form

$$
A=\left\langle v_{1}, \ldots, v_{n} \mid\left[v_{i}, v_{j}\right]=1 \forall(i, j) \in E\right\rangle .
$$

Thus $A$ is encoded by a graph with vertex set $\left\{v_{1}, \ldots, v_{n}\right\}$ and edge set $E \subset V \times V$. The prototype $F_{2} \times \cdots \times F_{2}$ is the RAAG associated to the 1-skeleton of the simplicial join $\mathbb{S}^{0} * \cdots * \mathbb{S}^{0}$. The Salvetti complex is the classifying space for $A$ obtained by gluing standard tori (cubes with opposite faces identified) along coordinate faces according to the commuting relations in the presentation; it has non-positive curvature.

I have portrayed right angled Artin groups (RAAGs) as a natural generalisation of direct products of free groups, but in many ways this fails to do them justice. They have gained prominence in recent years as an extremely important class of groups whose simple description belies their rich structure. From the point of view of this article, their three most important features are the richness of their subgroup structure, the ease with which they can be made to act on a great range of objects, and their residual properties.

6.1. RAAGS everywhere. Whenever one has $n$ automorphisms $\alpha_{i}$ of an object $X$, some of which commute, say $\left[\alpha_{i}, \alpha_{j}\right]=1$ if $(i, j) \in E$, then one has an action of the RAAG associated to the $n$-vertex graph with edge-set $E$. Roughly speaking, this action will be faithful if the $\alpha_{i}$ that do not commute are unrelated. One such setting is that of surface automorphisms: if two simple closed curves on a surface are disjoint, then the Dehn twists in those curves commute, but if one has a set curves no pair of which can be homotoped off each other, then suitable powers of the twists in those curves freely generate a free group. (Significantly sharper results of this sort are proved in [52] and [33].) It follows that any RAAG can be embedded in the mapping class group of any surface $S$ of sufficiently high genus: it suffices that the dual of the graph defining $A$ can be embedded in $S$. (This is explained by Crisp and Wiest in [35].) The surface can have boundary and punctures. With more care, one can arrange for the embedding of the RAAG to lie in the Torelli subgroup $\mathcal{T}(S)<\operatorname{Mod}(S)$ (cf. Koberda [52]).

Proposition 6.1. Every RAAG embeds in the mapping class group of any surface of sufficiently high genus.

6.2. Some Properties of RAAGs. With the preceding proposition in hand, we see that the theorems stated in Section 4 will follow if we can construct RAAGs with subgroups of the desired kind. Similarly, we shall solve Baumslag's problems (5.2) by constructing suitable RAAGs and exploiting the following theorem proved by Droms in his thesis [38] (cf. [39] and [67]).

Theorem 6.2. [38] RAAGS are residually torsion-free-nilpotent.

Further important properties of RAAGS include the fact that they are linear [49] even over $\mathbb{Z}$ [37]; they are conjugacy separable [58]; they are RFRS in the sense of Agol [2]; and their quasi-convex subgroups are virtual retracts (and so are closed in the profinite topology) [46]. 
6.3. Special cube complexes. What has really brought RAAGs to the fore in recent years is the richness of their subgroup structure. We saw hints of this in the work of Bestvina and Brady, but the spectacular extent of this richness truly emerged from the theory of special cube complexes initiated by Haglund and Wise [47] and advanced in many subsequent papers, particularly by Wise and his co-authors. (The lectures of Sageev have much more on this.)

Definition 6.3. A non-positively curved cube complex $X$ is special if it admits a locally isometric embedding into the Salvetti complex $K(A, 1)$ of a RAAG $A$.

Remark 6.4. A locally isometric map between compact non-positively curved spaces induces an injective map on fundamental groups ([24], p.201), so the fundamental groups of special cube complexes are subgroups of RAAGs.

The definition of special is not a very practical one, but Haglund and Wise [47] prove that it is equivalent to a short list of conditions on the behaviour of hyperplanes in the given cube complex (see Sageev's lectures).

Theorem 6.5. [47] A non-positively curved cube complex is special if and only if its hyperplanes are 2-sided, do not self-cross, do not self-osculate, and do not inter-osculate.

This remarkable insight makes it possible to verify specialness (of $X$ or some finite cover of it) in many instances. Thus we have a putative machine for constructing interesting subgroups of RAAGs (hence residually torsion-free nilpotent groups that are subgroups of mapping class groups):

- Cubulate groups, i.e. find methods for exhibiting large classes of groups as fundamental groups of compact non-positively curved cube complexes. (This is the central theme of Sageev's lectures.)

- Use the Haglund-Wise criterion to prove that these cube complexes $X$ are special, or at least that finite-sheeted covers of them are special (i.e. $X$ is virtually special).

This programme, widely promoted by Dani Wise, has proved extremely successful. Two results are of particular importance for our purposes, one for each of the steps articulated above. To state the first, we need the vocabulary of small cancellation groups.

The symmetrisation $R^{*}$ of a set of words $R$ over an alphabet $X$ consists of all cyclic permutations of $w$ and $w^{-1}$ with $w \in R$. The set $R$ and the presentation $\langle X \mid R\rangle$ are said to satisfy the $C^{\prime}(\lambda)$ small cancellation condition if pieces (i.e. recurring subwords) are bounded in length: if there exist distinct $w, w^{\prime} \in R^{*}$ such that $w \equiv u v$ and $w^{\prime} \equiv u v^{\prime}$, then $|u|<\lambda|w|$. If $R$ is finite and $m \geq 6$, then $\Gamma \cong\langle X \mid R\rangle$ is hyperbolic (in the sense of Gromov) and torsion-free ${ }^{3}$.

Theorem 6.6. [68] If a group $G$ has a finite presentation that is $C^{\prime}(1 / 6)$, then $G$ acts properly and cocompactly on a $\mathrm{CAT}(0)$ cube complex.

Many groups are now proved to be virtually special. The crowning achievment, following much work of Wise [69] and others, is Agol's theorem.

\footnotetext{
${ }^{3}$ In saying this, I'm skipping over a technicality about how to treat words in $R$ that are proper powers.
} 
Theorem 6.7. [1] Let $X$ be a compact non-positively curved cube complex. If $\pi_{1} X$ is hyperbolic, then $X$ is virtually special.

This theorem has many consequences - the most important to date being Agol's resolution of the virtual fibering conjecture for hyperbolic 3-manifolds (cf. Section 11)but the two that concern us here are the following.

Corollary 6.8. If a hyperbolic group $H$ is the fundamental group of a compact nonpositively curved cube complex, then $H$ embeds in the mapping class group of infinitely many (closed) surfaces, and some subgroup of finite-index $H_{0}<H$ is residually torsionfree nilpotent.

Proof. The first assertion follows from Proposition 6.1 and Corollary 2.2, the second from Theorem 6.2.

6.4. The mapping class genus of virtually special groups. Corollary 6.8 assures us that the following quantity, which one might call the mapping class genus, is a welldefined invariant of hyperbolic groups that can be cubulated. It seems difficult to compute but may well provide a rich field of exploration. The case of Kleinian groups is already intriguing. Here, $S_{g}$ denotes the closed orientable surface of genus $g$.

$$
\operatorname{mcg}(\Gamma):=\min \left\{g \mid \exists \Gamma \hookrightarrow \operatorname{Mod}\left(S_{g}\right)\right\} .
$$

\section{Rips, Fibre Products And 1-2-3}

We take up the theme of Construction 3.1. The lack of finite presentability in the fibre products that we constructed $P<F \times F$ can be traced to the fact that a non-trivial normal subgroup of infinite index in a free group cannot be finitely generated. The key to getting around this problem is to express groups as quotients of hyperbolic groups instead of free groups, with a gain in the finiteness properties of the kernel. This idea is due to E. Rips [62].

Theorem 7.1. [62] There is an algorithm that, given a finite group-presentation $\mathcal{Q}$, will construct a short exact sequence

$$
1 \rightarrow N \rightarrow H \stackrel{q}{\rightarrow} Q \rightarrow 1
$$

where $H$ is $C^{\prime}(1 / 6)$ small-cancellation, $Q$ is the group with presentation $\mathcal{Q}$, and $N$ is a 2-generator group.

Remark 7.2. The discussion in the previous section shows that $H$ is virtually special. In fact, one can arrange this more directly: the Rips construction is very flexible and variations by different authors have imposed extra conditions on $H$; before Agol's work, Haglund and Wise [47] used this flexibility to arrange for $H$ to be virtually special (at the cost of adding more generators to $N$ ).

In the spirit of Construction 3.1, we focus on the fibre product $P=\left\{\left(h, h^{\prime}\right) \in H \times H \mid\right.$ $\left.q(h)=q\left(h^{\prime}\right)\right\}$. We want $P$ to be finitely presented, but in general it will not be (cf. Lemma 7.7). However, if $Q$ is of type $F_{3}$ (i.e., has a classifying space $K(Q, 1)$ with finite 3 skeleton), then the 1-2-3 Theorem of [8] assures us that $P$ will be finitely presented. 
Theorem 7.3. [8] Let $1 \rightarrow N \rightarrow \Gamma \stackrel{q}{\rightarrow} Q \rightarrow 1$ be a short exact sequence of groups. If $N$ is finitely generated, $\Gamma$ is finitely presented, and $Q$ is of type $F_{3}$, then the associated fibre product $P<\Gamma \times \Gamma$ is finitely presented.

One may wonder if $N$ can also be made finitely presented, but it cannot.

Lemma 7.4. If $Q$ is infinite, then the subgroup $N<\Gamma$ in the Rips construction is not finitely presented.

Proof. Being a small cancellation group, $\Gamma$ has cohomological dimension 2. Bieri [13] proved that a finitely presented normal subgroup of infinite index in a group of cohomological dimension 2 must be free. But in the Rips construction $N$ is visibly not free: it is a 2-generator group that has non-trivial relations, and it is not cyclic because non-elementary hyperbolic groups do not have cyclic normal subgroups.

7.1. Finite and nilpotent quotients. Proposition 5.5 and its corollary show that if $Q$ has no finite quotients and $H_{2}(Q, \mathbb{Z})=0$ then the pair of groups $N \stackrel{u}{\rightarrow} \Gamma$ produced by the Rips construction is such that $u$ induces an isomorphism modulo each term of the lower central series, and so does the inclusion of the fibre product $P \hookrightarrow \Gamma \times \Gamma$. Likewise, Proposition 5.8 tells us that these inclusions induce isomorphisms of profinite completions.

7.2. Rips translates foibles from $Q$ to $N$ and $P$. The translation of properties from $Q$ to $N$ and $P$ has to be analysed according to context. The ones that interest us here concern finite and nilpotent quotients (as described above), decision problems and finiteness conditions.

If $Q$ has an unsolvable word problem, this manifests itself in unsolvable decision problems of a different type for $N$ and $P$.

Proposition 7.5. [8] Let $1 \rightarrow N \rightarrow \Gamma \stackrel{p}{\rightarrow} Q \rightarrow 1$ be a short exact sequence of groups, with $\Gamma$ finitely generated, and let $P<\Gamma \times \Gamma$ be the associated fibre product. If the word problem in $Q$ is unsolvable, then the membership problem for $P<\Gamma \times \Gamma$ is unsolvable.

Proof. We fix a finite generating set $X$ for $\Gamma$ and work with the generators $X^{\prime}=$ $\{(x, 1),(1, x) \mid x \in X\}$ for $\Gamma \times \Gamma$. Given a word $w=x_{1} \ldots x_{n}$ in the free group on $X$, we consider the word $\left(x_{1}, 1\right) \ldots\left(x_{n}, 1\right)$ in the free group on $X^{\prime}$. This word defines an element of $P$ if and only if $p(w)=1$ in $Q$, and we are assuming that there is no algorithm that can determine which words in the symbols $p(x)$ equal the identity in $Q$.

Proposition 7.6. [8] Let $1 \rightarrow N \rightarrow \Gamma \stackrel{p}{\rightarrow} Q \rightarrow 1$ be a short exact sequence of groups, with $\Gamma$ torsion-free and hyperbolic, and let $P<\Gamma \times \Gamma$ be the associated fibre product. If the word problem in $Q$ is unsolvable, then the conjugacy problem is unsolvable in $N$ and in $P$.

Sketch of proof: Fix finite generating sets $B$ for $\Gamma$ and $A$ for $N$. Fix $a \in N \backslash\{1\}$. For each $b \in B$ and $\epsilon= \pm 1$, let $u_{b, \epsilon}$ be a word in the free group on $A$ so that $b^{\epsilon} a b^{-\epsilon}=u_{b, \epsilon}$ in $H$. Given an arbitrary word $w$ in the letters $B$, one can use the relations $b^{\epsilon} a b^{-\epsilon}=u_{b, \epsilon}$ to convert $w a w^{-1}$ into a word $w^{\prime}$ in the letters $A$. Now ask if $w^{\prime}$ is conjugate to $a$ in $N$. The answer is "yes" if $w \in N$, and consideration of centralizers shows that it is "no" 
if $w \notin N$. Thus $w^{\prime}$ is conjugate to $a$ in $N$ if and only if $p(w)=1$ in $Q$, and we are assuming that there is no algorithm that can decide if this is the case.

The argument for $P$ is similar but more involved; see [8, Section 3].

The following lemma is proved using the LHS spectral sequence; see [29, Section 6].

Lemma 7.7. Let $1 \rightarrow N \rightarrow G \rightarrow Q \rightarrow 1$ be a short exact sequence of finitely generated groups. If $H_{3}(G, \mathbb{Q})$ is finite dimensional but $H_{3}(Q, \mathbb{Q})$ is infinite dimensional, then $\mathrm{H}_{2}(N, \mathbb{Q})$ is infinite dimensional.

\section{Examples Template}

The following template can be employed in any context where one is interested in demonstrating diverse or extreme behaviour among the finitely presented subgroups of groups in a class of groups $\mathcal{C}$ with the property that every RAAG embeds in some $\Gamma \in \mathcal{C}$.

- Feed designer groups $Q$ into the Rips construction to obtain

$$
1 \rightarrow N \rightarrow H \rightarrow Q \rightarrow 1 .
$$

- Pass to a subgroup of finite index in $H$ to obtain

$$
1 \rightarrow N_{0} \rightarrow H_{0} \stackrel{q}{\rightarrow} Q_{0} \rightarrow 1,
$$

$N_{0}$ finitely generated, $H_{0}$ special (subgroup of a $R A A G$ ) and $Q_{0}<Q$ finite-index.

- Pass to the fibre product

$$
P=\left\{\left(h, h^{\prime}\right) \in H_{0} \times H_{0} \mid q(h)=q\left(h^{\prime}\right)\right\}
$$

and note that $P$ is a subgroup of a RAAG.

- Note that by the 1-2-3 Theorem, $P$ is finitely presented ${ }^{4}$ if $Q$ is of type $F_{3}$.

- Embed the RAAG containing $P$ in $\Gamma \in \mathcal{C}$.

This is a rather general and loosely stated template, but it does have remarkably wide applicability. We'll use it to resolve the problems for mapping class groups and nilpotent genus that are the focus of our story. But to make use of the template one has to resolve the following difficulties:

- If you are interested in constructing finitely presented groups $P<H_{0} \times H_{0}$ with some property $\mathcal{P}$ then you must first identify a related property $\overline{\mathcal{P}}$ such that if $Q$ has $\overline{\mathcal{P}}$ then $P<H_{0} \times H_{0}$ has $\mathcal{P}$ (cf. subsection 7.2).

- You have to prove that there exist groups of type $F_{3}$ with property $\overline{\mathcal{P}}$.

- You have to ensure that $\overline{\mathcal{P}}$ is inherited by subgroups of finite index $Q_{0}<Q$; this might be inherent to $\overline{\mathcal{P}}$ but you might have to control the finite-index subgroups of $Q$, perhaps arranging that there are none other than $Q$ itself; cf. [21] and [23].

\footnotetext{
${ }^{4}$ If there is an algorithm to construct a finite 3-skeleton for a $K(Q, 1)$, then one can construct a finite presentation for $P$ in an algorithmic manner, but this requires further argument [26].
} 
8.1. Crafting designer groups. The construction of input groups $Q$ for the template is very particular to the situation at hand and typically requires ad hoc innovation. In order to prove Theorems 4.2, 4.3 and 5.3 we need groups with the following properties:

\section{Examples 8.1.}

(1) There are infinite groups of type $F_{3}$ that have no non-trivial finite quotients. The first such examples were constructed by Graham Higman [48] and a general method for constructing such groups is described in [21] and [23].

(2) There are groups of type $F_{3}$ that have an unsolvable word problem. Examples of this sort were constructed by Collins and Miller [34].

(3) There exist infinite groups $Q$ of type $F_{3}$ that, simultaneously, have no non-trivial finite quotients, have an unsolvable word problem, and $H_{2}(Q, \mathbb{Z})=0$; see $[22$, Theorem 3.1].

(4) There are finitely presented groups $\Delta$ with no non-trivial finite quotients, so that $H_{i}(\Delta, \mathbb{Z})=0$ for $i=1,2$ but $\operatorname{dim} H_{3}(\tilde{\Delta}, \mathbb{Q})=\infty$. Such groups are constructed in $[29]$.

\section{Proofs From the Template}

Proposition 6.1 and Corollary 2.2 tell us that Theorems 4.3 and 4.2 will follow if we can prove the same results for subgroups of RAAGs or virtually special groups. Likewise, since RAAGs are residually torsion-free-nilpotent, in order to prove Theorem 5.3 it is enough to exhibit RAAGs with the stated properties. In each case, we shall use the Examples Template to construct suitable RAAGs. I shall state the key points and I encourage the reader to check the details.

9.1. Proof of Theorems 4.3 and 4.2. Let property $\overline{\mathcal{P}}$ be the insolubility of the word problem. Apply the template with $Q$ as in Example 8.1(2) and appeal to Propositions 7.5 and 7.6 .

9.2. Proof of Theorem 5.3(1). Let property $\overline{\mathcal{P}}$ be the insolubility of the word problem. Apply the template with $Q$ as in Example 8.1(3) and appeal to Corollary 5.6 and Proposition 7.6.

9.3. Proof of Theorem 5.3(2). Apply the template with $Q$ as in Example 8.1(4) and appeal to Proposition 5.5 and Lemma 7.7.

\section{The Isomorphism Problem for Subgroups of RAAGs and $\operatorname{Mod}(S)$}

To prove Theorem 4.1 we use a criterion due to Bridson and Miller [27].

Theorem 10.1. [27] Let $1 \rightarrow N \rightarrow \Gamma \rightarrow L \rightarrow 1$ be an exact sequence of groups. Suppose that

(1) $\Gamma$ is torsion-free and hyperbolic,

(2) $N$ is infinite and finitely generated, and

(3) $L$ is a non-abelian free group. 
If $F$ is a non-abelian free group, then the isomorphism problem for finitely presented subgroups of $\Gamma \times \Gamma \times F$ is unsolvable.

In more detail, there is a recursive sequence $\Delta_{i}(i \in \mathbb{N})$ of finite subsets of $\Gamma \times \Gamma \times F$, together with finite presentations $\left\langle\Delta_{i} \mid \Theta_{i}\right\rangle$ of the subgroups they generate, such that there is no algorithm that can determine whether or not $\left\langle\Delta_{i} \mid \Theta_{i}\right\rangle \cong\left\langle\Delta_{0} \mid \Theta_{0}\right\rangle$.

The subgroup $G_{i}$ presented by $\left\langle\Delta_{i} \mid \Theta_{i}\right\rangle$ is obtained by fixing a splitting $\Gamma=N \rtimes L$ and defining $G_{i}$ to be the subgroup generated by $N \times N$ and $\left\{\left(\phi_{i}(x), x\right) \mid x \in F\right\}$ where $\phi_{i}: F \rightarrow L \times L$ is a homomorphism whose image is a subdirect product. A key feature of the construction in [27] is that the finiteness properties of centralisers in $G_{i}$ are intimately connected to the question of whether $\phi_{i}$ is onto. We saw in Section 3 that there is no algorithm that can determine if a finite subset of a direct product of free groups generates the product, and this provides a seed of undecidability that propogates through the construction.

Corollary 10.2. There exist RAAGs in which the isomorphism problem for finitely presented subgroups is unsolvable.

Proof. We apply the template of Section 8 with $Q$ a non-abelian free group and define $\Gamma=H_{0}$. If $H_{0}$ is a subgroup of the RAAG $A$, then $\Gamma \times \Gamma \times F$ will be a subgroup of the RAAG $A \times A \times F$.

In the light of Proposition 6.1, Theorem 4.1 follows from this corollary.

Remark 10.3. Baumslag and Miller [9] proved that the isomorphism problem is unsolvable in the class of finitely presented residually torsion-free-nilpotent groups. Corollary 10.2 provides an alternative proof.

\section{DeHn FUnCTIONS}

One can prove Theorem 4.4 without the Rips construction or the 1-2-3 Theorem: one can deduce it directly from the fact that the fundamental groups of closed hyperbolic 3 -manifolds are virtually special, applying Proposition 6.1 to embed the RAAG $A$ of the following proposition into mapping class groups.

Proposition 11.1. There exist right-angled Artin groups $A$ and finitely presented subgroups $P<A$ such that $P$ has an exponential Dehn function.

Proof. Let $M$ be a closed, orientable, hyperbolic 3-manifold that fibres over the circle. Then $\pi_{1} M=\Sigma \rtimes \mathbb{Z}$ where $\Sigma$ is the fundamental group of a closed surface of genus at least 2 , and $\Gamma=\pi_{1} M \times \pi_{1} M$ contains $P:=(\Sigma \times \Sigma) \rtimes \mathbb{Z}$, the inverse image of the diagonal in $\Gamma /(\Sigma \times \Sigma)=\mathbb{Z} \times \mathbb{Z}$. The Dehn function of $P$ is exponential; see [19] Theorem 2.5. The growth of a Dehn function is preserved on passage to subgroups of finite index, and by [1] there is a subgroup of finite index in $\Gamma$ that embeds in a right-angled Artin group.

\section{REFERENCES}

[1] I. Agol, The virtual Haken conjecture (with appendix by I. Agol, D. Groves and J. Manning), Documenta Math. 18 (2013) 1045-1087.

[2] I. Agol, Criteria for virtual fibering, J. Topol. 1 (2008), 269-284.

[3] S. Andreadakis, On the automorphisms of free groups and free nilpotent groups, Proc. London Math. Soc. 15 (1965) 239-268. 
[4] Matthias Aschenbrenner, Stefan Friedl, Henry Wilton, 3-Manifold groups,arXiv:1205.0202.

[5] H. Bass and A. Lubotzky, Linear-central filtrations on groups, In "The mathematical legacy of Wilhelm Magnus: groups, geometry and special functions (Brooklyn NY, 1992)", Contemp. Math., vol. 169, pp. 45-98. Amer. Math. Soc., Providence, RI, 1994.

[6] G. Baumslag, Musings on Magnus, In "The mathematical legacy of Wilhelm Magnus: groups, geometry and special functions (Brooklyn NY, 1992)", Contemp. Math., vol. 169, pp. 99-106. Amer. Math. Soc., Providence, RI, 1994.

[7] G. Baumslag, Parafree groups, in Infinite groups: geometric, combinatorial and dynamical aspects, 1-14, Progr. Math., 248, Birkhäuser, (2005).

[8] G. Baumslag, M. R. Bridson, C. F. Miller III and H. Short, Fibre products, non-positive curvature, and decision problems, Comment. Math. Helv. 75 (2000), 457-477.

[9] G. Baumslag and C.F. Miller III, The isomorphism problem for residually torsion-free nilpotent groups, Groups Geom. Dyn. 1 (2007), 1-20.

[10] G. Baumslag and J. Roseblade, Subgroups of direct products of free groups, J. London Math. Soc. (2) 30 (1984) 44-52.

[11] M. Bestvina and N. Brady, Morse theory and finiteness properties of groups, Invent. Math. 129 (1997), 445-470.

[12] R. Bieri, Homological dimension of discrete groups, Queen Mary College Mathematics Notes (1976).

[13] R. Bieri, Normal subgroups in duality groups and in groups of cohomological dimension 2, J. Pure Appl. Algebra 7 (1976), 35-51.

[14] J.S. Birman, A. Lubotzky and J. McCarthy, Abelian and solvable subgroups of the mapping class groups, Duke Math. J. 50 (1983), 1107-1120.

[15] N. Brady, M. Clay, Matt and P. Dani, Morse theory and conjugacy classes of finite subgroups, Geom. Dedicata 135 (2008), 15-22.

[16] M.R. Bridson, On the subgroups of right angled Artin groups and mapping class groups, Math. Res. Lett. 20 (2013), 1-10.

[17] M.R. Bridson, Non-positive curvature and complexity for finitely presented groups, International Congress of Mathematicians. Vol. II, 961-987, Eur. Math. Soc., Zurich 2006.

[18] M.R. Bridson, The rhombic dodecahedron and semisimple actions of $\operatorname{Aut}\left(F_{n}\right)$ on $\mathrm{CAT}(0)$ spaces, Fund. Math. 214 (2011), 13-25.

[19] M. R. Bridson, On the subgroups of semihyperbolic groups, in "Essays on geometry and related topics", pp. 85-111, Monogr. Enseign. Math. 38, Geneva, 2001.

[20] M.R. Bridson, Finiteness properties for subgroups of $G L(n, \mathbf{Z})$, Math. Ann. 317 (2000), 629-633.

[21] M. R. Bridson, Controlled embeddings into groups that have no non-trivial finite quotients, Geometry and Topology Monographs 1 (1998), Epstein Birthday Schrift, (Rivin, Rourke, Series, eds.), 99-116.

[22] M. R. Bridson, Decision problems and profinite completions of groups, J. Algebra 326 (2011), 59-73.

[23] M. R. Bridson and F.J. Grunewald Grothendieck's problems concerning profinite completions and representations of groups, Ann. of Math. (2) 160 (2004), 359-373.

[24] M. R. Bridson and A. Haefliger, "Metric Spaces of Non-Positive Curvature", Grund. Math. Wiss. 319, Springer-Verlag, Heidelberg-Berlin, 1999.

[25] M. R. Bridson, J. Howie, C. F. Miller III and H. Short, Subgroups of direct products of limit groups. Ann. of Math. 170 (2009), 1447-1467.

[26] M. R. Bridson, J. Howie, C. F. Miller III and H. Short, On the finite presentation of subdirect products and the nature of residually free groups. Amer. J. Math., 135 (2013), 891-933.

[27] M. R. Bridson and C. F. Miller III, Recognition of subgroups of direct products of hyperbolic groups, Proc. Amer. Math. Soc., 132 (2003), 59-65.

[28] M. R. Bridson and C. F. Miller III, Structure and finiteness properties of subdirect products of groups. Proc. London Math. Soc. (3) 98 (2009), 631-651.

[29] M. R. Bridson and A. W. Reid, Nilpotent completions of groups, Grothendieck pairs, and four problems of Baumslag, arXiv:1211.0493

[30] M. R. Bridson and R. D. Wade, Actions of higher rank lattices on free groups, Compositio Math., 147 (2011), 1573-1580. 
[31] K. S. Brown, "Cohomology of groups", Graduate Texts in Mathematics 87, Springer-Verlag, BerlinHeidelberg-New York (1982).

[32] P-E. Caprace, Lectures on proper CAT(0) spaces and their isometry groups, this volume.

[33] M. Clay, C. Leininger and J. Mangahas. The geometry of right angled Artin subgroups of mapping class groups, To appear in Groups Geom. Dyn.

[34] D.J. Collins and C.F. Miller III, The word problem in groups of cohomological dimension 2, Groups St. Andrews 1997 in Bath I (ed. C. M. Campbell, Robertson and Smith), LMS Lecture Notes 260 (Cambridge University Press, Cambridge, 1999) 211-218.

[35] J. Crisp and B. Wiest. Quasi-isometrically embedded subgroups of braid and diffeomorphism groups. Trans. Amer. Math. Soc. 359 (2007), 5485-5503.

[36] F. Dahmani, V. Guirardel and D. Osin, Hyperbolically embedded subgroups and rotating families in groups acting on hyperbolic spaces, arXiv:1111.7048

[37] M. W. Davis and T. Januszkiewicz, Right-angled Artin groups are commensurable with right-angled Coxeter groups, J. Pure Appl. Algebra, 153 (2000), 229-235.

[38] C. Droms, Graph Groups (Algebra, Kim, Roush, Magnus), PhD Thesis, Syracuse University, 1983.

[39] G. Duchamp and D. Krob, The lower central series of the free partially commutative group, Semigroup Forum 45 (1992), 385-394.

[40] B. Farb, Some problems on mapping class groups and moduli space. In Problems on Mapping Class Groups and Related Topics (B. Farb, editor), Proceedings of symposia in pure mathematics, vol 74 . American Math. Soc., Providence RI, 2006, pp. 11-56.

[41] B. Farb, A. Lubotzky and Y. Minsky, Rank-1 phenomena for mapping class groups, Duke Math. J. 106 (2001), 581-597.

[42] B. Farb and D. Margalit, A Primer on Mapping Class Groups, Princeton Univ. Press, Princeton NJ, 2012.

[43] B. Farb and H. Masur, Superrigidity and mapping class groups, Topology 37 (1998), 1169-1176.

[44] E.K. Grossman, On the residual finiteness of certain mapping class groups, J. London Math. Soc.9 (1974), 160-164.

[45] F.J. Grunewald, On some groups which cannot be finitely presented, J. London Math Soc. 17 (1978), 427-436.

[46] F. Haglund, Finite index subgroups of graph products, Geom. Dedicata 135 (2008), 167-209.

[47] F. Haglund and D.T. Wise, Special cube complexes, Geom. Funct. Anal. 17 (2008), 1551-1620.

[48] G. Higman, A finitely generated infinite simple group, J. London Math Soc. 26 (1951), 61-64.

[49] S.P. Humphries, On representations of Artin groups and the Tits conjecture, J. Algebra 169 (1994), $847-862$.

[50] N. Ivanov, Subgroups of Teichmüller Modular Groups. Translations of Mathematical Monographs, vol. 115, Amer. Math. Soc., Providence RI, 1992.

[51] S.P. Kerckhoff, The Nielsen realization problem, Ann. of Math. 117 (1983), 235-265.

[52] T. Koberda. Right-angled Artin groups and a generalized isomorphism problem for finitely generated subgroups of mapping class groups. Preprint 2012, arXiv:1007.1118.

[53] R. Kulkarni, Symmetries of surfaces, Topology 26 (1987), 195-203.

[54] J. McCarthy, A "Tits-alternative" for subgroups of surface mapping class groups, Trans. Amer. Math. Soc. 291 (1985), 583-612.

[55] C. F. Miller III, "On group-theoretic decision problems and their classification", Ann. Math. Studies, No. 68, Princeton University Press (1971).

[56] C. F. Miller III, Decision problems for groups: survey and reflections, in Algorithms and classification in combinatorial group theory, MSRI Publ., vol 23, SpringerVerlag, New York, 1992, pp. 1-59.

[57] K. A. Mihailova, The occurrence problem for direct products of groups, (Russian) Dokl. Akad. Nauk SSSR 119 1958, 1103-1105.

[58] A. Minasyan, Hereditary conjugacy separability of right angled Artin groups and its applications, Groups Geom. Dyn. 6 (2012), 335-388.

[59] G. Mislin, Classifying spaces for proper actions of mapping class groups, Münster J. Math. 3 (2010), 263-272.

[60] L. Mosher, Mapping class groups are automatic, Ann. of Math. (2) 142 (1995), 303-384. 
[61] V. P. Platonov and O. I. Tavgen, Grothendieck's problem on profinite completions and representations of groups, K-Theory 4 (1990), 89-101.

[62] E. Rips, Subgroups of small cancellation groups, Bull. London Math Soc. 14 (1982), 45-47.

[63] M. Sageev, Codimension-1 subgroups and splittings of groups, this volume.

[64] J. R. Stallings, Homology and central series of groups, J. Algebra 2 (1965), 170-181.

[65] J.R. Stallings, A finitely presented group whose 3-dimensional integral homology is not finitely generated, Amer. J. Math. 85 (1963), 541-543.

[66] R.D. Wade, Johnson homomorphisms and actions of higher-rank lattices on right-angled Artin groups, J. London Math. Soc., to appear, arXiv:1101.2797

[67] R.D. Wade, The lower central series of a right-angled Artin group, arXiv:1109.1722

[68] D. T. Wise, Cubulating small cancellation groups, GAFA, 14(1):150-214, 2004.

[69] D. T. Wise, The Structure of Groups with a Quasiconvex Hierarchy, preprint, McGill 2011.

Martin R. Bridson, Mathematical Institute, Andrew Wiles Building, Oxford OX2 6GG, U.K.

E-mail address: bridson@maths.ox.ac.uk 